\title{
KONZEN, Paulo Roberto. O conceito de liberdade de imprensa ou de liberdade de comunicação pública na filosofia do direito de G. W. F. Hegel. Porto Alegre: Editora Fi, 2013. 462p.
}

\section{* Horacio Martín Sisto}

\begin{abstract}
A primera vista el tema abordado por este libro podría sugerir que el 1 - autor se ha concentrado en un aspecto muy limitado de la filosofía de Hegel, como es la libertad de imprenta y la libertad de comunicación pública. Pues bien, a medida que el lector se adentra en la obra, puede apreciar que el autor desarrolla el tema como un punto clave y certero en donde converge no sólo la concepción política de Hegel en su conjunto, sino también elementos fundamentales de su concepción metafísica y antropológica. Es por ello que Paulo Konzen comienza el examen desde la relación entre categorías metafísicas como diversidad e igualdad, considera luego la relación entre individuo y la comunidad de la que forma parte, hasta investigar el problema de la libertad y sus instanciaciones jurídicas concretas.

Un concepto que recorre todo el primer capítulo es el de diversidad humana. Este capítulo presenta una tensa oscilación entre una igualdad que se les reconoce a los hombres, en el plano del derecho abstracto, y una diversidad que acusa la naturaleza y que da lugar a una serie de desigualdades en el ámbito de la sociedad civil. El autor recorre varios aspectos de esta tensión y llega a una primera solución. Según ella, Hegel busca una integración que en cuanto tal se distancie del individualismo disolvente, pero que a su vez no se transforme en una homologación
\end{abstract}

* Doctor en Filosofía. Profesor Adjunto de Filosofía Moderna en la Universidad Nacional de General Sarmiento (UNGS) <msisto@ungs.edu.ar>. Jefe de Trabajos Prácticos de Filosofía de la Historia en la Universidad de Buenos Aires (UBA) < martin_sisto@filo.uba.ar>. 
en la pura identidad, es decir la anulación de la diferencia. Konzen logra mostrar claramente en el pensamiento de Hegel la preocupación por la integración. En cambio, la diversidad llegaría solo a mostrarse como algo "no negativo". Hasta allí sería difícil convencer a quien objetase la disolución de la significación del individuo en la comunidad. Las diferencias serían aceptadas, sí, pero como mera particularidad, no significativa. Pero esta primera formulación se enriquece luego con la elucidación del concepto de "Estado orgánico". Explorando la analogía con el organismo, Konzen muestra diversos aspectos por los cuales la diversidad no sólo es un factor a tolerar sino elemento indispensable y funcional al todo. Un organismo viene a menos, incluso puede morir si le falta alguna de sus partes o una de ellas no cumple su función. No obstante, aun así podría objetarse qué tipo de libertad le resta a quien está atado en su significación a una función necesaria, y a qué tipo de unidad se somete toda diversidad en términos funcionales. Después de todo, el organismo es una metáfora; se trata de ver con más precisión cuáles son las implicaciones conceptuales que Hegel está dispuesto a aceptar. Creo que el autor acierta en la delimitación de esta integración y de la metáfora del organismo. La cuestión fundamental que preocupa a Hegel es que la diversidad humana, que puede manifestarse en muchas direcciones y en diversos grados, no someta a su arbitrariedad mediante la generación de desigualdades cada vez mayores, tanto materiales como culturales, el destino de quienes no se encuentran favorecidos por la naturaleza o por la formación. Lejos de buscar anular la diversidad, que como hombre de humanidades es particularmente sensible a la formación y los diversos talentos, como lo muestra Konzen a través de varias citaciones, Hegel acepta limitar el ámbito de esta diversidad sólo en la medida en que pueda llevar a una desigualdad que atente directamente a los menos provistos material y espiritualmente, e indirectamente a la consistencia de la nación o pueblo como un todo, del cual a través de distintas mediaciones abrevan, al fin y al cabo, incluso los talentos y las formaciones destacadas.

Finalmente el autor muestra como en diversos contextos Hegel ha expresado su rechazo hacia aquellos gobiernos que intentan engañar al pueblo, por lo cual una interpretación del valor que tiene para Hegel la libertad de imprenta debe contar con el rechazo de Hegel de cualquier tipo de manipulación posible de un pueblo por parte de un gobierno.

Sin embargo, Konzen ya adelanta que para que un pueblo pueda tener un discernimiento propio y correcto de la cuestión pública, lo cual es un derecho, es necesario que tenga la mayor formación (Bildung) posible. La libertad de comunicación favorece la mediación de la diversidad, en particular a través de su efecto sobre la opinión pública, como veremos luego en su relación con la formación. 
La reducción de la noción de libertad de prensa como "libertad de decir y escribir lo que se quiere" es obra del formalismo, análoga a la reducción de la noción de la libertad a "hacer lo que se quiere"; el autor, atento a esta advertencia que Hegel lanza al comienzo de la observación § 319, tomará como cuestión central la elucidación del concepto de libertad de comunicación, en lugar de darla por obvia y limitar la exposición sólo al dilema de si Hegel defendía o no la libertad de imprenta. En el segundo capítulo, entonces, se adentra en la noción de libertad misma y en la importancia que tiene la Bildung para la realización de la libertad del hombre, a fin de mostrar luego cómo la publicidad colabora para dicha formación. Para ello expone al comienzo la recepción que hace Hegel de la noción de estado de naturaleza según Hobbes, noción que Hegel admite como contrafigura de la libertad. La voluntad sólo es imputable en la medida en que ha tenido oportunidad de acceso al conocimiento, en particular de la ley; la falta de formación implica la eventual reducción de un sector de la población al estado de naturaleza, es decir una situación en donde el hombre no es libre y es amenaza y hasta efectiva eliminación de la vida de sus semejantes, y esto sin imputabilidad alguna. En las complejas relaciones entre el querer y el saber, la formación permite al hombre tener un discernimiento correcto. Es por ello que la publicidad es esencial para todos los integrantes de la comunidad. La formación, gracias al saber y a la información de la que se nutre, permite mediar el interés particular con el universal, de modo que la voluntad no padece así lo universal como una imposición externa o alienante, sino como parte aceptada de sí. El autor muestra mediante el examen de la los diversos aspectos de la Bildung en la Filosofía del Derecho, cómo el pasaje del estado de naturaleza a la Eticidad, es decir de la no libertad a la libertad, no es un salto puramente político en sentido estrecho, ni un salto de la voluntad por la cual ella reprime su interés en función de un universal externo, sino que tiene su sendero interno y constitutivo en la formación de la voluntad individual, desde las instancias de la familia hasta el Estado. En esta - me atrevo a traducir - pedagogía política de la voluntad, juega un rol esencial la publicidad, la libertad de comunicación, que en la sociedad moderna se encuentra vinculada con la libertad de imprenta (Konzen, atento al texto del § 319 y también al contexto histórico, admite prácticamente como sinónimos libertad de comunicación pública y libertad de imprenta).

Se trata de formación o trabajo de la cultura (Bildung), que involucra tanto el trabajo cultural personal del individuo como de toda la sociedad, dentro de la cual se encuentra la comunicación y la imprenta. El autor explora también la incidencia de la Bildung en el ámbito de la sociedad civil, por ejemplo en cómo afecta en la participación en el patrimonio en 
lo que respecta a la formación profesional; y en el ámbito del Estado, por ejemplo en lo que concierne a la competencia e idoneidad de los funcionarios. De la articulación compleja de estas instancias depende el grado de cultura de una nación. Según Konzen, Hegel sostiene al respecto una suerte de círculo virtuoso, pues la libertad de comunicación pública es uno de los mejores medios para la formación cultural de un pueblo y por ende de su capacidad de discernimiento, y a su vez, a mayor grado de cultura, reina con más probabilidad un mayor grado de libertad de comunicación pública, pues es el menos probable el abuso de la libertad en cuestión.

El autor no se limita al examen de los textos y recorre en el capítulo tercero las vicisitudes biográficas e históricas en donde los textos hegelianos fueron ideados, escritos y publicados. Así sale a la luz un Hegel que fue periodista en Bamberg y sintió directa (mientras fue director) e indirectamente la censura (cuando el periódico fue clausurado, poco tiempo después de haber renunciado a su cargo). Pero sobre todo el capítulo se articula sobre la problemática que presentan los Principios de la Filosofía del Derecho, en cuanto obra sorprendida por las Resoluciones de la Convención de Karlsbad poco tiempo antes de publicarse y que se enfrenta con la paradoja de hablar de la libertad de prensa en una época de censura. Konzen afronta el examen de las posiciones principales un arco extremo de intérpretes, desde los que sostienen que los Principios de la Filosofía del Derecho son la consagración filosófica del gobierno prusiano por parte de un Hegel condescendiente, hasta quienes sostienen una dicotomía tajante entre el Hegel de la obra y el de las Lecciones; un arco que recorre numerosas variantes. Pero aquí el autor debe afrontar el problema no de si la censura afectó una de las tantas tesis de la filosofía del derecho, sino justamente a la idea misma de la libertad de comunicación pública. En el examen de las interpretaciones, Konzen encuentra la ocasión para ir presentando al lector su propia posición y sus razones. Aun cuando concede que en particular el §319 y su anotación son confusos, por momentos más bien alusivos y elípticos, sostiene que existe una esencial continuidad entre el Hegel de Bamberg y de las Lecciones de Filosofía del Derecho de 1817 y siguientes, por un lado, y la obra publicada, los Principios de la Filosofía del Derecho, por el otro.

La exposición sistemática del concepto de libertad de comunicación pública y de libertad de imprenta, y la ponderación de sus alcances y límites encuentran lugar en el capítulo final, el cuarto. Mediante un análisis exegético de los elementos decisivos del §319 y su Anotación, determina sucesivamente los elementos fundamentales del concepto. Puesto que gran parte de la exposición hegeliana procede allí mediante negación - se extiende más en señalar cuándo existe abuso de libertad 
de comunicación que en ofrecer una exposición en términos positivos - Konzen se sirve metodológicamente del dictum que Hegel evoca de Spinoza - toda determinación es negación - para detectar por contraste en cada instanciación de abuso que Hegel señala, el uso legítimo admitido implícitamente. Luego de un extenso relevamiento y confrontación con la bibliografía sobre el tema, el autor presenta una reconstrucción descriptiva de los elementos fundamentales de la libertad de comunicación pública. Y sostiene que la concepción hegeliana de esta instancia de la libertad converge con la que presentan las principales realidades constitucionales de los estados contemporáneos. El autor presenta, entre otros, los siguientes elementos argumentativos. Hegel sin duda no es un defensor de la libertad de imprenta en términos de libertad absoluta. Admite que puedan considerarse ilícitos los abusos. Pero en primer lugar exige una ley; los alcances y límites de la libertad de comunicación pública no pueden depender del mero arbitrio del gobierno. En cuanto a los abusos, siguiendo gran parte de la bibliografía, Konzen destaca cómo los casos que presenta Hegel son más bien casos en donde la libertad de comunicación pública es utilizada como medio para cometer otro delito ya tipificado como tal en general en los códigos, y con entidad autónoma, como ser por ejemplo calumnia e injurias, o la incitación a la revuelta. Por otra parte, el texto presenta una salvaguarda explícita de la libertad de la ciencia, que recordemos que para Hegel incluye a la filosofía como su mayor exponente. Por lo demás, en los casos donde la expresión pública puede ser potencialmente un riesgo, por ejemplo, para la seguridad de la nación, señala que debe considerarse si efectivamente ese riego existe, pues una expresión es sólo peligrosa si el contexto lo justifica. Luego, en todo lo que atañe a la aplicación de sanciones, Konzen subraya cómo Hegel es más bien restrictivo en los casos dudosos, y deja más bien a la misma opinión pública a que los regule con sus propias sanciones, como el desprecio y la atribución de insignificancia.

Pero la reconstrucción que realiza Konzen de los fundamentos en los dos primeros capítulos, no sólo sienta las bases para su posición acerca de la libertad de imprenta en Hegel, sino que también le permite trascender el contexto histórico puntual. La necesidad humana esencial de la formación, en saber y discernimiento, que requiere como uno de sus medios mejores, según Hegel, a la libertad de comunicación pública, puede según Konzen sugerir criterios para la situación actual. En líneas generales, nos puede proveer de criterios para pensar los nuevos problemas que nos acechan hoy, a diferencia de ese entonces, como ser la sobreabundancia y el exceso de información; el aplanamiento de toda información al mismo nivel de importancia, y por tanto la necesidad de un concepto crítico de información en función de la libertad 
humana, cuestiones hoy medulares en todo planteo de ética y legislación comunicacional. Así también la obligación que exige Hegel del Estado de respetar el ámbito de la libertad subjetiva y la privacidad, limitaría según Konzen por ejemplo la posible injerencia en los correos electrónicos, y exigiría una fundamentación muy estricta de una ley que autorizase en algunos casos su intervención.

Este libro presenta el rigor de una tesis doctoral, sobre la cual se basó originalmente, sin quedar encerrado en el carácter críptico que presentan a veces los textos académicos - sobre todo cuando se trata de Hegel - para el lector que proviene de otras ramas de la filosofía o bien de otras disciplinas como la ciencia política. A lo largo de su investigación, Konzen presenta una paciente y minuciosa labor filológica que recorre con atención el texto de la Filosofía del Derecho y las Lecciones publicadas en diversas ediciones críticas, y considera con el peso debido a los agregados (Zusätze). Considera a la Filosofía hegeliana del Derecho dentro de una lectura directa del resto de la obra de Hegel, en la cual se encuentra el Espíritu Objetivo y articula y atiende continuamente a la terminología en lengua original. El autor lee también los textos hegelianos a partir del trabajo realizado por sus predecesores, con una especial atención a las publicaciones de sus colegas en Brasil, con lo cual acerca al público latinoamericano, como es el caso de quien escribe, no sólo sus propias reflexiones y conclusiones, sino también toda la importante labor que se viene efectuando desde hace años en los círculos brasileños de estudiosos de la obra de Hegel.

\section{Endereço postal:}

Universidad de Buenos Aires (UBA)

Puán 480 - Barrio de Cabalito

Ciudad Autónoma de Buenos Aires, Argentina

Data de recebimento: 10/01/2014

Data de aceite: 30/02/2014 